\title{
Incorporating Extrinsic Goals Into Decision and Cost-Effectiveness Analyses
}

\author{
Gordon B. Hazen, PhD, Alan Schwartz, PhD
}

It has not been widely recognized that medical patients as individuals may have goals that are not easily expressed in terms of quality-adjusted life years ( $Q A L Y s$ ). The QALY model deals with ongoing goals such as reducing pain or maintaining mobility, but goals such as completing an important project or seeing a child graduate from college occur at unique points in time and do not lend themselves to easy expression in terms of QALYs. Such extrinsic goals have been posited as explanations for preferences inconsistent with the QALY model, such as unwillingness to trade away time or accept gambles. In this article, the authors examine methods for including extrinsic goals in medical decision and cost-effectiveness analyses. As illustrations, they revisit 2 previously published analyses, the management of unruptured intracranial arteriovenous malformations (AVMs) and the evaluation of preventive strategies for BRCA+women. Key words: theories of quality of life; utility inconsistencies; utility measurement; multiattribute utility function; health state preferences, utilities, and valuations; healthrelated quality of life; cancer prevention. (Med Decis Making $X X X X ; X X: X X-X X)$
$\mathbf{M}$ ethods for evaluating health quality are central to medical decision analyses and cost-effectiveness analyses. The most important such method is the quality-adjusted life year (or QALY) model, ${ }^{1}$ in which a patient's survival duration in a health state is weighted by a quality coefficient proportional to the quality of health the patient experiences. The recommendation of the Panel on Cost Effectiveness in Health and Medicine ${ }^{2}$ is that medical cost-effectiveness studies should incorporate morbidity and mortality consequences into a single measure using QALYs. QALYs have indeed become ubiquitous in these and other analyses.

However, as Tsevat ${ }^{3}$ points out, numerous studies have demonstrated that the correlation between one's current health and the quality coefficient for a health state elicited by time-trade-off or standard gamble techniques is at best modest. Willingness to

Received 9 January 2008 from the Department of Industrial Engineering and Management Sciences, Northwestern University, Evanston, Illinois (GBH) and Department of Medical Education, University of IIlinois, Chicago (AS). Revision accepted for publication 26 November 2008.

Address correspondence to Gordon B. Hazen, PhD, Department of Industrial Engineering and Management Sciences, Northwestern University, Evanston, IL 60208; e-mail: hazen@iems.northwestern.edu.

DOI: 10.1177/0272989X09333121 trade away time or take a gamble is often much less than the general public, health care professionals, and even family members believe.

One of us ${ }^{4}$ has previously suggested that unwillingness to trade away time or take a gamble may be due to the unacknowledged presence of goals that are extrinsic to the QALY model. Such extrinsic goals differ from ongoing goals, such as pain reduction or mobility, which can be addressed via quality coefficients and have a cumulative effect dependent on life duration. Instead, extrinsic goals such as completing a project or seeing a child graduate from college are not ongoing but are achieved at specific points in time. For extrinsic goals, the level of goal achievement has importance that is unrelated to life duration. In a telephone survey by Schwartz and others, $^{5} 50$ community members revealed 232 extrinsic goals involving education, family, health and fitness, personal fulfillment, professional issues, travel, and wealth, and a survey of 101 inpatients revealed an additional 459 extrinsic goals in the same categories. However, reports of extrinsic goals are not new: in discussing maximum endurable time preferences in 1998, Miyamoto and others ${ }^{6}$ mention an individual who wanted to live 5 years to see his son graduate from high school. Pliskin and others $^{1}$ in 1980 mention a respondent who felt obligated to prolong his life as long as he could function as a father and provider for his family. 
Hazen $^{4}$ presents preference axioms that yield an extension of the QALY model that includes extrinsic goals. As Hazen shows, this extension can, in principle, account for empirical violations of the QALY model, such as the maximum endurable time phenomenon, ${ }^{6-9}$ or for participants' lack of willingness to trade away time for quality or take a gamble on improved quality that might shorten life. ${ }^{10}$ Schwartz and others ${ }^{5}$ report that community members were willing to trade off some (unspecified) amount of life for $48 \%$ of reported extrinsic goals, and inpatients were willing to do so for $58 \%$. Trading off time for quality at the expense of an extrinsic goal might therefore be plausibly declined. Among extrinsic goals, the greatest willingness to trade life years was in exchange for family-related goals.

In an empirical study, van der Pol and Shiell ${ }^{11}$ claim to find only limited support for the effect of extrinsic goals on time trade-offs when the extrinsic goal hypothesis is strictly interpreted as a target life expectancy. In our view, however, these authors have overlooked the adequate-state interpretation of the extrinsic goal hypothesis in which a goal is achieved not by surviving for a target duration but by spending a sufficient amount of time in health states that are adequate for goal achievement. The latter interpretation is, we believe, consistent with the observations they report. The application we treat below is of this type, where the extrinsic goal of bearing children-or of remaining in adequate (fertile) health states long enough to do so-may be contravened by the need for prophylactic oophorectomy (surgical removal of the ovaries) in women at high risk for ovarian cancer. This conflict is difficult or impossible to model acceptably under the QALY format.

The purpose of this article is to discuss how extrinsic goals might be incorporated into medical decision and cost-effectiveness analyses. We reanalyze 2 specific published medical decision analyses and explore the impact of plausible associated extrinsic goals. Along the way, we introduce goalachieved life years (GALYs), an extension of QALYs, to account for the impact of extrinsic goal achievement. The next section begins by presenting the extrinsic goal model from Hazen. ${ }^{4}$

\section{QALYs AND EXTRINSIC GOALS}

Hazen $^{4}$ provides a simple model for combining QALYs with extrinsic goals. If $h$ is a health profilethat is, a function that specifies for each point $t$ in time an individual's health state-and $g$ is the degree of extrinsic goal achievement associated with health profile $h$, then overall utility $U(g, h)$ is given by a weighted sum of QALYs and goal utility:

$$
U(g, h)=Q A L Y(h)+k_{G} U_{G}(g) .
$$

Here, QALY $(h)$ is the QALYs associated with health profile $h$, and $U_{G}(g)$ is the utility associated with goal achievement level $g$, normalized so that goal utility is 0 for the worst possible level $g_{0}$ of goal achievement and 1 for the best possible level $g^{*}$ :

$$
U_{G}\left(g_{0}\right)=0, U_{G}\left(g^{*}\right)=1 .
$$

The quantity $k_{G}$ is a positive weight equal to the number of full-health life years one would sacrifice to increase goal utility from its worst level, $U_{G}=0$, to its best level, $U_{G}=1$.

Plausible values of $k_{G}$ have begun to be established by empirical research. In a yet unpublished study of 52 outpatients by Schwartz and others, ${ }^{12}$ respondents identified their most salient extrinsic goal that could affect medical decision making and performed a time trade-off for life years with goal achievement v. life years without goal achievement. Results were as follows: of 52 outpatients ages 20 to 50 years old, 23 were willing to trade off all but 6 months of life for a self-selected extrinsic goal. Among the other 29, the mean and median willingness to trade was $50 \%$ of the remaining life years to which the individual aspired (95\% confidence interval $[\mathrm{CI}]: .37, .65)$. In raw units, this represented a mean willingness to trade 19 life years (95\% CI: 12, 26) for goal achievement. A conjoint analysis based on these data found that goal achievement was, on average, considered 1.9 times as preferable as 5 years of life.

An alternate representation equivalent to (1) is

$$
G A L Y(g, h)=Q A L Y(h)-k_{G} \cdot\left(1-U_{G}(g)\right),
$$

where the GALY term stands for goal-achieved life years and arises from the following hypothetical time-trade-off exercise. Assume for the moment that there is no time discounting, so that the QALYs associated with duration $t$ in optimal health $q^{*}$ are simply $t$, that is, QALY $\left(q^{*}, t\right)=t$. Suppose one regards the combination $(g, h)$ of health profile $h$ and goal achievement level $g$ as equivalent in preference to a shorter lifetime $t^{-}$in optimal health $q^{*}$ with goal $g^{*}$ fully achieved-that is, equivalent to $\left(g^{*}, q^{*}, t^{-}\right)$. For example, one might regard the health profile $h$ 
of spending 20 years in partial health with goal $g=$ unachieved as equivalent to spending $t^{-}=10$ years in full health $q^{*}$ with goal $g^{*}=$ achieved. This equivalence implies

$$
\operatorname{GALY}(g, h)=\operatorname{GALY}\left(g^{*}, q^{*}, t^{-}\right),
$$

and using (3) on the right side of this equation, we get

$$
\operatorname{GALY}(g, h)=Q A L Y\left(q^{*}, t^{-}\right)-k_{G}\left(1-U_{G}\left(g^{*}\right)\right) .
$$

But using $U_{G}\left(g^{*}\right)=1$ and $\operatorname{QALY}\left(q^{*}, t\right)=t$, this turns into

$$
\operatorname{GALY}(g, h)=t^{-},
$$

that is, GALY $(g, h)$ is equal to the equivalent number $t^{-}$of goal-achieved life years in optimal healthhence the term $G A L Y$. If time is discounted, then $\operatorname{GALY}(g, h)$ would equal the equivalent number of discounted goal-achieved life years in optimal health.

Several forms for the goal utility function $U_{G}$ are possible, depending on the context. Consider, for instance, the simple case in which goal achievement is binary (yes or no) and occurs only if survival duration $t_{h}$ of the health profile $h$ exceeds a critical duration $t_{G}$. Then we would have

$$
U_{G}(g)=F_{G}\left(t_{h}\right),
$$

where $F_{G}$ is a step function at $t_{G}$, yielding a utility of 1 for $t_{h} \geq t_{G}$ and 0 for $t_{h}<t_{G}$. If there is uncertainty about the critical time $t_{G}$, then $F_{G}\left(t_{h}\right)$ could be taken as the probability $\mathrm{P}\left(t_{h} \geq t_{G}\right)$ that the extrinsic goal is achieved by time $t_{h}$, in which case, $F_{G}$ would be the cumulative distribution function of $t_{G}$. In either case, expected goal utility is the probability that survival time $t_{h}$ exceeds $t_{G}$, that is,

$$
E U_{G}=P\left(t_{h}>t_{G}\right) .
$$

More generally, it might be (as mentioned above) that a goal can be achieved only from some set $A$ of adequate health states, and goal achievement occurs only if duration $t_{A}$ in adequate states of health exceeds a critical level $t_{G}$. Then,

$$
U_{G}(g)=F_{G}\left(t_{A}\right),
$$

where again $F_{G}$ could be a step function at $t_{G}$ or the cumulative distribution function of $t_{G}$ if it is uncertain.
In this case, expected utility is the probability that duration $t_{A}$ in adequate states exceeds $t_{G}$, that is,

$$
E U_{G}=P\left(t_{A}>t_{G}\right) .
$$

We shall consider these types of goal utility in the examples below.

\section{A SIMPLE EXAMPLE WHERE EXTRINSIC GOALS MATTER}

When might an extrinsic goal make a difference compared to a standard analysis using only QALYs? One possibility is that it may induce delay in treatment interventions. Consider, for instance, an analysis of the artificially simple situation in which an individual with suboptimal quality of life $q_{0}$ can select an intervention that would increase quality of life by an amount $\Delta q$. However, the intervention has immediate mortality risk $p_{M}>0$. The intervention does not extend lifetime, which we take to be a fixed duration $L$.

Based on QALY calculations, the intervention should be undertaken now or never. This is easy to see because if intervention is delayed until a time $t_{d}<L$, then overall expected QALYs are

$$
\begin{aligned}
E[Q A L Y]= & Q A L Y \text { s up to time } t_{d} \\
& +p_{M}(\text { zero additional } Q A L Y s) \\
& +\left(1-p_{M}\right)\left(\text { additional } Q A L Y s \text { after time } t_{d}\right) \\
= & q_{0} t_{d}+\left(1-p_{M}\right)\left(q_{0}+\Delta q\right)\left(L-t_{d}\right) .
\end{aligned}
$$

As a function of delay time $t_{d}$, E[QALY] decreases if the mortality odds are less than a critical ratio:

$$
\frac{p_{M}}{1-p_{M}} \leq \frac{\Delta q}{q_{0}},
$$

in which case it is optimal to intervene immediately. Otherwise, it is optimal to delay intervention until the end of life, that is, to never intervene.

We focus on the former case, that is, when immediate intervention is QALY optimal. Consider what happens if there is now an extrinsic goal to survive until time $t_{G}<L$. Then goal utility is given by

$$
\mathrm{E} U_{G}=\mathrm{P}\left(t_{h}>t_{G}\right)= \begin{cases}1-p_{M} & \text { if } t_{d}<t_{G} \\ 1 & \text { if } t_{d} \geq t_{G}\end{cases}
$$

Under our extrinsic goal model (3), expected GALY as a function of delay $t_{d}$ is given by 


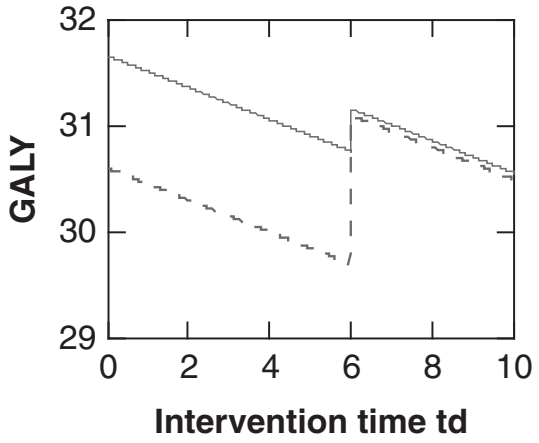

$$
\mathrm{kG}=2 \mathrm{yr}-\cdots \cdot \mathrm{kG}=7 \mathrm{yr}
$$

Figure 1 Expected goal-achieved life years (GALYS) as a function of intervention time $t_{d}$ when extrinsic goal importance $k_{G}$ is 2 years and 7 years. Delaying intervention until time $t_{G}=6$ years is optimal if the extrinsic goal is sufficiently important $\left(k_{G}=7\right.$ years).

$$
\begin{aligned}
E[G A L Y] & =E[Q A L Y]-k_{G}\left(1-E U_{G}\right) \\
& =\mathrm{E}[Q A L Y]-k_{G} \begin{cases}p_{M} & \text { if } t_{d}<t_{G} \\
0 & \text { if } t_{d} \geq t_{G} .\end{cases}
\end{aligned}
$$

Therefore, expected GALY jumps higher by an amount $k_{G} p_{M}$ when intervention time $t_{d}$ reaches $t_{G}$. If $k_{G}$ is large enough, the jump in overall utility at $t_{d}=t_{G}$ will be large enough to make delaying until time $t_{G}$ optimal. The situation is shown in Figure 1 when $t_{G}=6$ years, $p_{M}=0.2, q_{0}=0.65, \Delta q=0.35$.

The conclusion, obvious in retrospect, is that the presence of a sufficiently important extrinsic survival time goal may make it optimal to delay an intervention until the extrinsic goal is achieved, even if a QALY analysis prescribes immediate intervention.

\section{THE POTENTIAL DELAY OF RISKY SURGERY}

We consider a decision analysis performed by Auger and Weibers ${ }^{13}$ concerning the management of unruptured intracranial arteriovenous malformations (AV$\mathrm{Ms}$ ). At the time of the analysis, some authorities favored elective excision of the AVM before it ruptures, whereas others advised watchful waiting-nonintervention unless rupture occurs - at which time surgical excision would be performed in those who survive. Auger and Weibers formulated a discrete-time Markov model comparing these 2 alternatives and found that expected QALYs for 38-year-old patients is greater for immediate elective surgery. They found an improvement in expected QALYs of $8.8 \%$-that is, $14.8-13.6=1.2$ years for a 38-year-old patient.

We wished to explore the option of delaying elective surgery until an arbitrary time $t_{d} \geq 0$ in the presence of an extrinsic goal. To facilitate this, we reformulated Auger and Weibers's model as a continuous-time Markov chain, displayed in Figure 2 as a factored stochastic tree. ${ }^{14-16}$ In this model, the option of delaying elective surgery until time $t_{d}$ includes the possibility that by time $t_{d}$, it may be too late for elective surgery-the patient may already have died or already had emergency surgery due to AVM rupture. Quality coefficients are the same as in Auger and Weibers and are displayed in the Surgery component in Figure 2. The discount rate we used, $r=5 \%$, is also the same.

We added an extrinsic goal represented by a survival duration surrogate of $t_{G}=6$ years. Expected goal utility $\mathrm{E} U_{G}$ is then the probability of surviving 6 years or more. This quantity is shown in Figure 3 as a function of elective surgery intervention time $t_{d}$. $\mathrm{E} U_{G}$ decreases from a probability 0.923 of 6-year survival at $t_{d}=0$ to a probability 0.889 of 6 -year survival for $t_{d}$ just under 6 years, the decrease due to the increasing likelihood of an AVM rupture before surgery can remove the AVM. As $t_{d}$ crosses from below 6 years to above it, $\mathrm{E} U_{G}$ jumps up to a probability 0.940 of 6-year survival, the jump of 0.051 due to the fact that surgical mortality $p_{D}=0.059$ can no longer prevent 6-year survival. (The jump is less than $p_{D}$ because it may be too late for surgery at time $t_{d}$.)

Because goal utility at $t_{d}=6$ years exceeds goal utility at any other $t_{d}$ less than 6 years, it is optimal in terms of goal utility to delay elective surgery until $t_{d}=6$ years. However, quality-adjusted life years may also be important. Figure 4 shows expected QALYs and overall expected goal-achieved life years $\mathrm{E}[\mathrm{GALY}]=\mathrm{E}[\mathrm{QALY}]-k_{G}\left(1-\mathrm{E} U_{G}\right)$ as a function of intervention time $t_{d}$. Expected QALYs decrease from a value of 14.3 years at $t_{d}=0$ to an asymptotic value of 13.4 years as $t_{d}$ approaches infinity, indicating the QALY optimality of immediate elective surgery over watchful waiting, a conclusion consistent with Auger and Weibers. ${ }^{13}$

At a value of $k_{G}=10$ years (goal achievement is worth 10 years of life), expected GALYs decrease from 13.6 years at $t_{d}=0$ to 12.8 years for $t_{d}$ just below 6 years and then jump to 13.2 years at $t_{d}=6$ years, not as large as the GALYs at $t_{d}=0$. So in terms of GALYs, immediate elective surgery is still optimal. The critical value of the goal weight $k_{G}$ above 


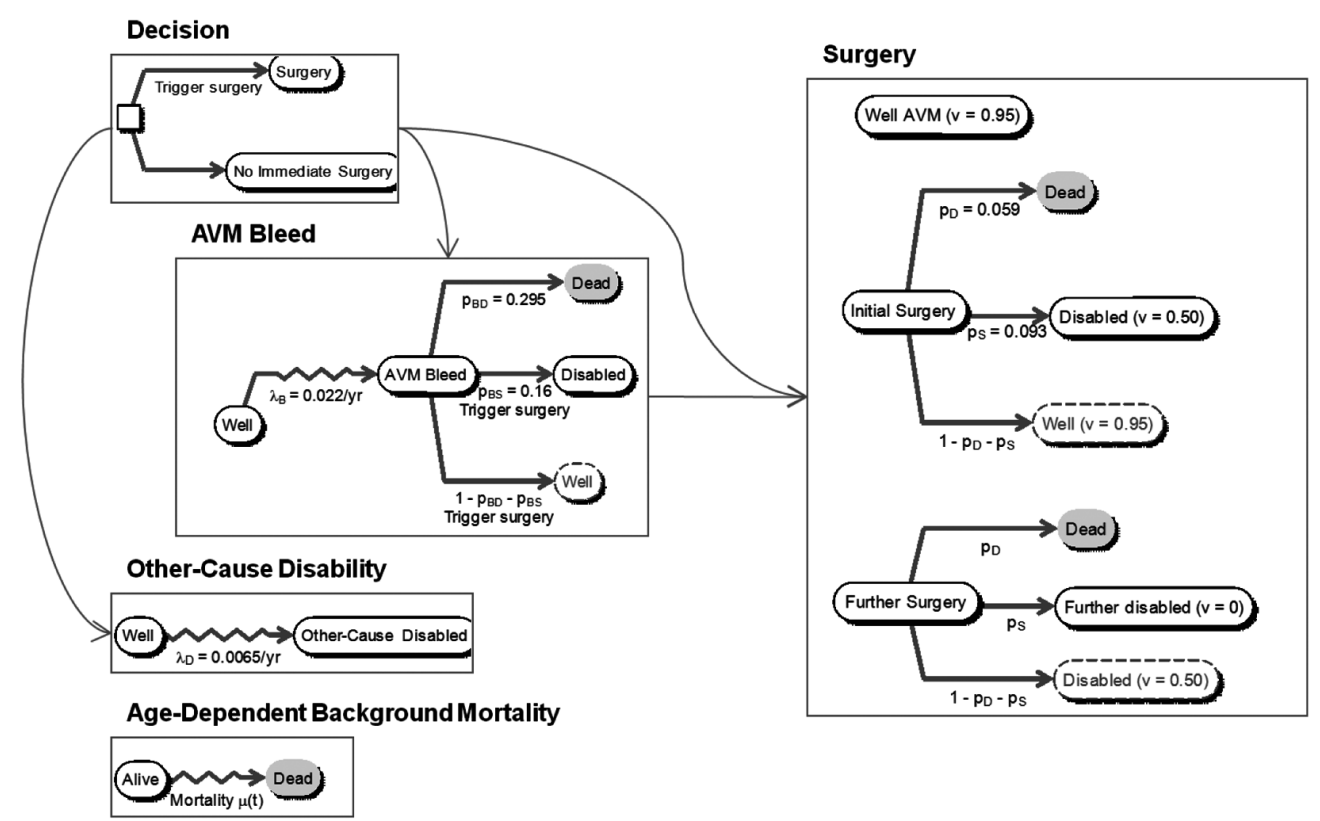

Figure 2 A continuous-time Markov model of the choice between elective and reactive surgery for arteriovenous malformation (AVM), formulated as a stochastic tree. Wavy arrows connecting nodes denote transitions that take time to occur and are labeled with rates (e.g., a transition rate of $\lambda_{B}=0.022$ year from Well to AVM Bleed in the AVM component of the model). Straight arrows connecting nodes denote immediate transitions and are labeled with probabilities (e.g., the probability $p_{D}=0.059$ of transition from Initial Surgery to Dead in the Surgery component). Curved arrows connecting model components indicate influence (e.g., the Surgery decision triggers a transition from Well AVM to Initial Surgery in the Surgery component).

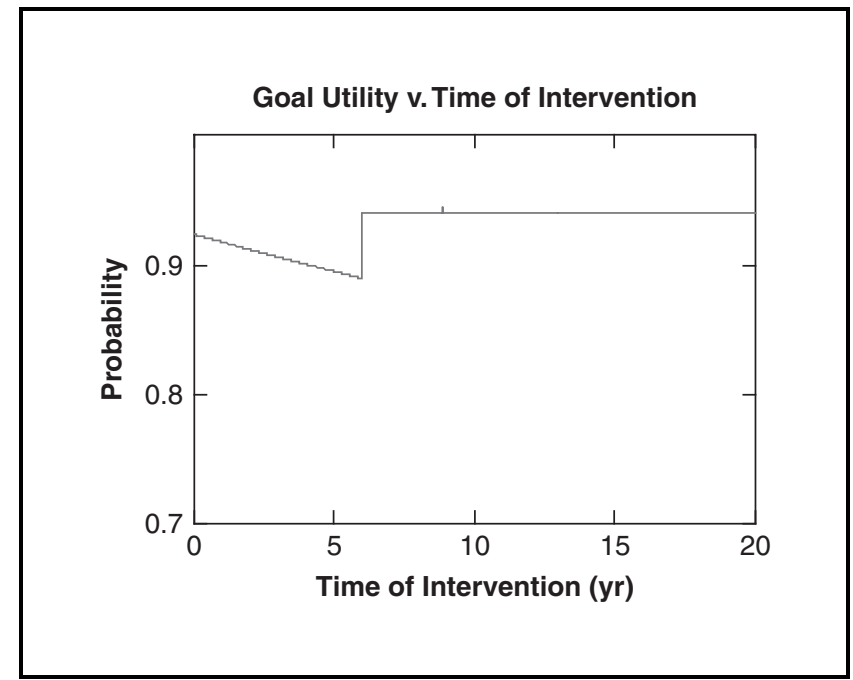

Figure 3 Expected goal utility, equal to the probability of 6 -year survival, as a function of intervention time $t_{d}$ for the decision problem of Figure 2.

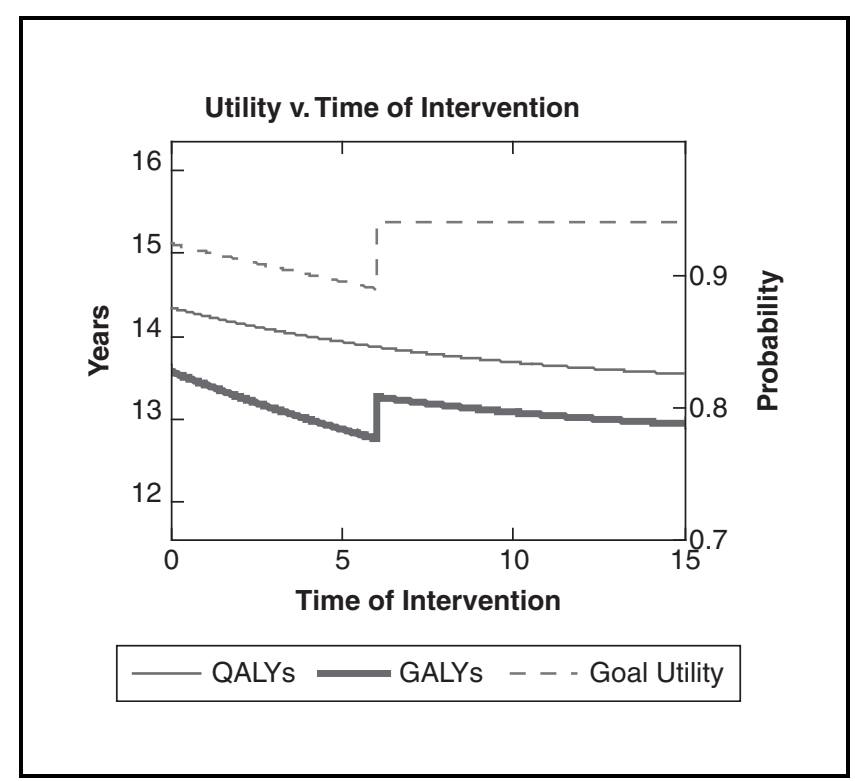

Figure 4 Expected quality-adjusted life years ( $Q A L Y$ S), expected goal-achieved life years (GALYs), and expected goal utility as a function of intervention time $t_{d}$ for the model of Figure 2. 
which watchful waiting until $t_{G}$ is GALY optimal is $k_{G}=28.2$ years. This value seems large but is still within the range of responses reported by Schwartz and others, ${ }^{12}$ as mentioned above. So for at least some of those respondents, watchful waiting until $t_{G}=6$ years would be the preferred alternative.

\section{PREVENTIVE STRATEGIES FOR BRCA + WOMEN}

Women carrying BRCA1 or BRCA2 mutations are at high risk for breast and ovarian cancers at young ages. Anderson and others ${ }^{17}$ conducted a costeffectiveness analysis of preventive strategies for such women. Strategies considered were tamoxifen, oral contraceptives, simple surveillance, prophylactic mastectomy, prophylactic oophorectomy (removal of the ovaries), and the combination of prophylactic mastectomy and oophorectomy. The results for a 35-year-old BRCA1 woman were that the 2 strategies, mastectomy + oophorectomy and oophorectomy, dominated (more life years at less cost) all other strategies, with mastectomy + oophorectomy having a favorable incremental cost-effectiveness ratio of \$2352 per life year over oophorectomy. Comparable results held when life years were quality adjusted and for BRCA2 women.

Oophorectomy is, of course, not a feasible strategy for women still desiring to bear children, and Anderson and others' results ${ }^{17}$ are not applicable to this subpopulation. To remedy this, it would be possible to conduct a separate cost-effectiveness (CE) analysis for this subpopulation, omitting oophorectomy and mastectomy strategies and perhaps adding strategies such as delayed oophorectomy/mastectomy. However, the omission of strategies may distort incremental CE ratios for the remaining strategies. Childbearing is an extrinsic goal, and one can argue that the right approach is to use GALYs to correctly downwardly adjust the utility of oophorectomy or mastectomy for this class of women. We report here the results adapting Anderson and others' analysis in this way. For simplicity, we consider here only BRCA1 carriers.

We wished to explore the option of delaying oophorectomy until an arbitrary time $t_{d} \geq 0$, in the presence of an extrinsic childbearing goal. To facilitate this, we constructed a continuous-time Markov model based on Anderson and others' assumptions and data. ${ }^{17}$ This allows us to vary $t_{d}$ continuously in a cohort analysis instead of the more demanding Monte Carlo analysis performed by Anderson and others.
Because cancer mortality post diagnosis is not time stationary, it cannot be directly represented in a stationary Markov model. We therefore reformulated the breast cancer, ovarian cancer, and endometrial cancer components of the analysis by Anderson and others ${ }^{17}$ as cure rate models, ${ }^{18}$ which do have stationary Markov representations. The breast and ovarian cancer components are shown as stochastic trees in Figure 5. We computed maximum likelihood estimates of the cure and mortality rate parameters in these models from Surveillance Epidemiology and End Results (SEER) data. ${ }^{19}$

For BRCA-positive women having the extrinsic goal of bearing children, one plausible form for goal utility is (4), in which it is desired to remain in a set $A$ of adequate (in this case, fertile) health states for a goal duration $t_{G}$. For this model, the set $A$ is all states not involving oophorectomy, mastectomy, contraceptive use, any type of cancer, or death. We took the goal duration $t_{G}$ to be 2 years for a 35 -year-old woman. We took the goal weight $k_{G}$ in (1) to be 3 years-that is, goal achievement is worth 3 years of life in full health.

Our life year and cost results are shown in Figure 6 and Table 1 (discount rate 3\% as in Anderson and others ${ }^{17}$ ). These differ to some degree from Anderson and others.* However, the qualitative conclusions are very close: oophorectomy + mastectomy dominates all other strategies, and oophorectomy ranks second among strategies originally considered by Anderson and others. Because the extrinsic childbearing goal is not included, strategies involving a delay until $t_{G}$ (2 years) are dominated by the corresponding undelayed strategies.

However, in terms of goal-achieved life years, the cost-effectiveness results are quite different, as shown in Figure 7 and Table 2. GALYs for the 2 best undelayed strategies of Figure 7 are 3 years $\left(=k_{G}\right)$ less than the life years (LYs) of Figure 6 , and the originally dominant strategy, oophorectomy + mastectomy, no longer dominates, although it remains undominated. Two other undominated strategies emerge: oophorectomy delayed until $t_{G}$ and oophorectomy + mastectomy delayed until $t_{G}$. At a $\$ 50,000 /$ GALY cutoff, the latter is optimal, and the former is a close second.

\footnotetext{
*There are many possible reasons, including our use of continuoustime rather than discrete-time modeling, our use of cure rate models for cancer mortality, our omission for simplicity of cataract side effects under tamoxifen, and our use of time-stationary incidence rates where Anderson and others ${ }^{17}$ varied these by decade. Other differing features not fully documented in Anderson and others may be present as well.
} 


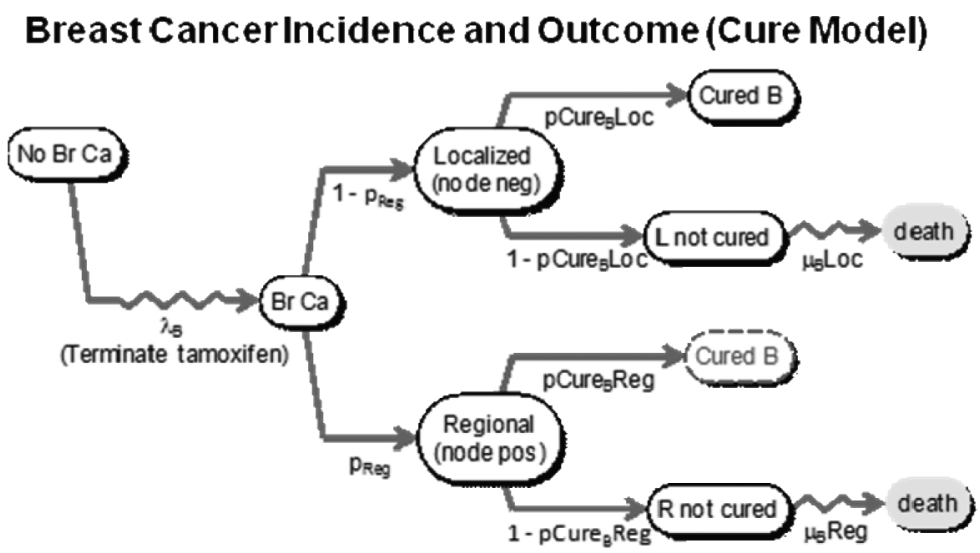

Ovarian Cancer Incidence and Outcome (Cure Model)

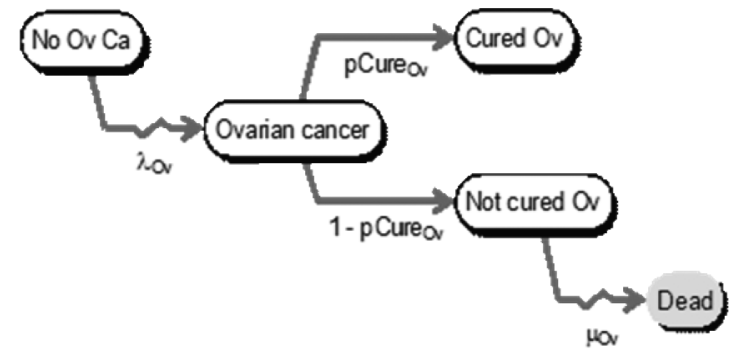

Figure 5 Stochastic trees depicting 2 components of our reformulation of Anderson and others ${ }^{17}$ as a continuous-time Markov chain. Both of these components are cure rate models fit from Surveillance Epidemiology and End Results (SEER) data. See Figure 2 for an interpretation of diagram features.

Observe that the naive approach of delaying the cost/LY optimal strategy, oophorectomy + mastectomy, until goal achievement time $t_{G}$ does in this case yield the cost/GALY optimal strategy. In general, however, this heuristic may mislead as delay may affect costs in differing ways across strategies-as in this case, delay has reversed the cost ranking between oophorectomy + mastectomy and oophorectomy.

One might also wonder whether a simpler cost/LY analysis with the undelayed strategies removed would be adequate. Indeed, with the value $k_{G}=3$ years that we have assumed for the goal importance weight, the incremental C/E ratio $\$ 2420$ of oophorectomy + mastectomy delayed over oophorectomy delayed (Table 2) is in fact equal to what would be obtained in this simpler cost/LY analysis, and this simpler analysis would reach the same conclusion that oophorectomy + mastectomy delayed is optimal.
However, for smaller values of $k_{G}$, this approximate equivalence fails-for example, when $k_{G}$ falls below 1.3 years in the current cost/GALY analysis, the undelayed strategy oophorectomy + mastectomy once again dominates. In general, performing a cost/LY analysis with extrinsic goal-impeding alternatives eliminated is roughly equivalent to setting goal importance $k_{G}$ to a large value in a cost/GALY analysis. The rough equivalence will be lost for smaller values of $k_{G}$. So in general, it seems safer to account for extrinsic goals using GALYs.

\section{CONCLUSION}

We have illustrated how extrinsic goals can be incorporated into medical decision and cost-effectiveness analyses. The approach we used requires 
Table 1 Costs, Life Years (LYs), and Cost-Effectiveness Ratios for All Strategies in Our Reanalysis of Anderson and others ${ }^{17}$ for a 35-Year-Old BRCA1 Woman

\begin{tabular}{|c|c|c|c|c|c|}
\hline Strategy & Cost, US\$ & $\Delta$ Cost, $\mathbf{U S}^{\mathbf{a}}$ & $\mathbf{L Y}$ & $\Delta \mathbf{L} \mathbf{Y}^{\mathbf{a}}$ & $\Delta \operatorname{Cost} / \Delta \mathbf{L Y}$ \\
\hline Oophorectomy + mastectomy & 115,185 & 0 & 23.9 & 0 & - \\
\hline Oophorectomy & 119,554 & 4369 & 22.5 & -1.4 & Dominated \\
\hline Oophorectomy delayed until $t_{G}$ & 126,649 & 11,464 & 22.1 & -1.8 & Dominated \\
\hline Oophorectomy + mastectomy delayed until $t_{G}$ & 128,510 & 13,325 & 22.9 & -1.1 & Dominated \\
\hline Tamoxifen & 146,283 & 31,097 & 20.2 & -3.7 & Dominated \\
\hline Oral contraceptives & 147,879 & 32,694 & 21.9 & -2.0 & Dominated \\
\hline Mastectomy & 150,435 & 2556 & 22.5 & -1.4 & Dominated \\
\hline Oral contraceptives delayed until $t_{G}$ & 152,133 & 36,947 & 21.5 & -2.5 & Dominated \\
\hline Mastectomy delayed until $t_{G}$ & 154,735 & 39,550 & 21.8 & -2.1 & Dominated \\
\hline Surveillance & 162,379 & 47,193 & 20.5 & -3.5 & Dominated \\
\hline
\end{tabular}

All LYs and costs discounted at $3 \%$. Oophorectomy + mastectomy is the dominant strategy.

${ }^{\mathrm{a}}$ Compared to the next least expensive undominated alternative.

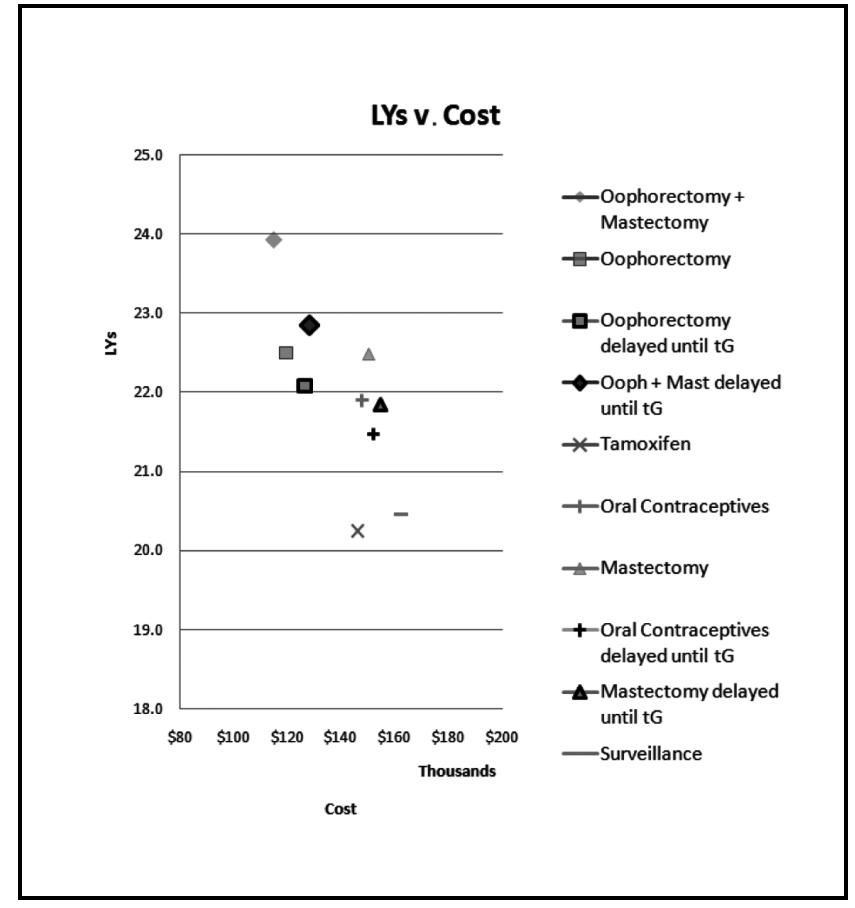

Figure 6 The cost-life years ( $L Y S$ ) plane for our reanalysis of Anderson and others ${ }^{17}$ for a 35-year-old BRCA1 woman. Oophorectomy + mastectomy dominates all other strategies. Identically shaped markers represent pairs of strategies, the darker bordered strategy delayed from its partner by $t_{G}=2$ years. See Table 1 for detailed costs and LYs.

the analyst to assess or draw on plausible reported values for the goal importance weight $k_{G}$ and to include an estimate $t_{G}$ of the time required for goal achievement. In many cases, it would be more realistic to treat $t_{G}$ as an uncertain quantity and specify its probability distribution or, better yet, to explicitly treat underlying goal achievement, for which $t_{G}$ is a surrogate. For example, our BRCA reanalysis presented here could be extended by exploiting fertility data to estimate the rate of achievement of successful pregnancy among fertile women. However, such a refinement would not substantially alter the form of the analyses we have presented.

As we have discussed, the QALY model by itself can only account for ongoing goals whose cumulative effect is proportional to life duration via quality coefficients. Our extrinsic goal or GALY model allows an individual's utility function to include a broader class of goals. An unfortunate point of confusion may arise because many sources refer to the quality coefficients in the QALY model as utilities. Properly speaking, these quantities are only rates of ongoing utility accrual, which only generate utility when multiplied by duration, and even then only utility for ongoing goals. The notion of utility is broader and can incorporate issues the QALY model fails to address such as those we have discussed in this article.

As our BRCA reanalysis suggests, extrinsic goals can be incorporated into cost-effectiveness policy analyses as well as individual patient analyses. GALYs would replace QALYs as the measure of effectiveness. The key ingredient would be an estimate of a population mean goal weight $\bar{k}_{G}$. Only the mean value is necessary for the same reason that only population mean quality coefficients are required for QALY computation-the GALY model is linear in $k_{G}$, and the population uncertainty in $k_{G}$ can reasonably be taken to be independent of the level of goal achievement, which it multiplies. 
Table 2 Goal-Achieved Life Years (GALYs) and Cost-Effectiveness Ratios for All Strategies in Our Reanalysis of Anderson and others ${ }^{17}$

\begin{tabular}{lllrr}
\hline \hline Strategy & Goal U & GALY & $\Delta$ GALY $^{\mathbf{a}}$ & $\Delta$ Cost $/ \Delta$ GALY \\
\hline Oophorectomy + mastectomy & 0 & 20.9 & 0 & - \\
Oophorectomy & 0 & 19.5 & -1.4 & Dominated \\
Oophorectomy delayed until $t_{G}$ & 0.8198 & 21.5 & 0.6 & $\$ 18,419$ \\
Oophorectomy + mastectomy delayed until $t_{G}$ & 0.8198 & 22.3 & 0.8 & $\$ 2420$ \\
Tamoxifen & 0.839 & 19.8 & -2.5 & Dominated \\
Oral contraceptives & 0 & 18.9 & -3.4 & Dominated \\
Mastectomy & 0 & 19.5 & -2.8 & Dominated \\
Oral contraceptives delayed until $t_{G}$ & 0.8198 & 20.9 & -1.4 & Dominated \\
Mastectomy delayed until $t_{G}$ & 0.8198 & 21.3 & -1.0 & Dominated \\
Surveillance & 0.8198 & 19.9 & -2.4 & Dominated \\
\hline
\end{tabular}

Oophorectomy + mastectomy, oophorectomy + mastectomy delayed, and mastectomy are the dominant strategies.

${ }^{\mathrm{a}}$ Compared to the next least expensive undominated alternative.

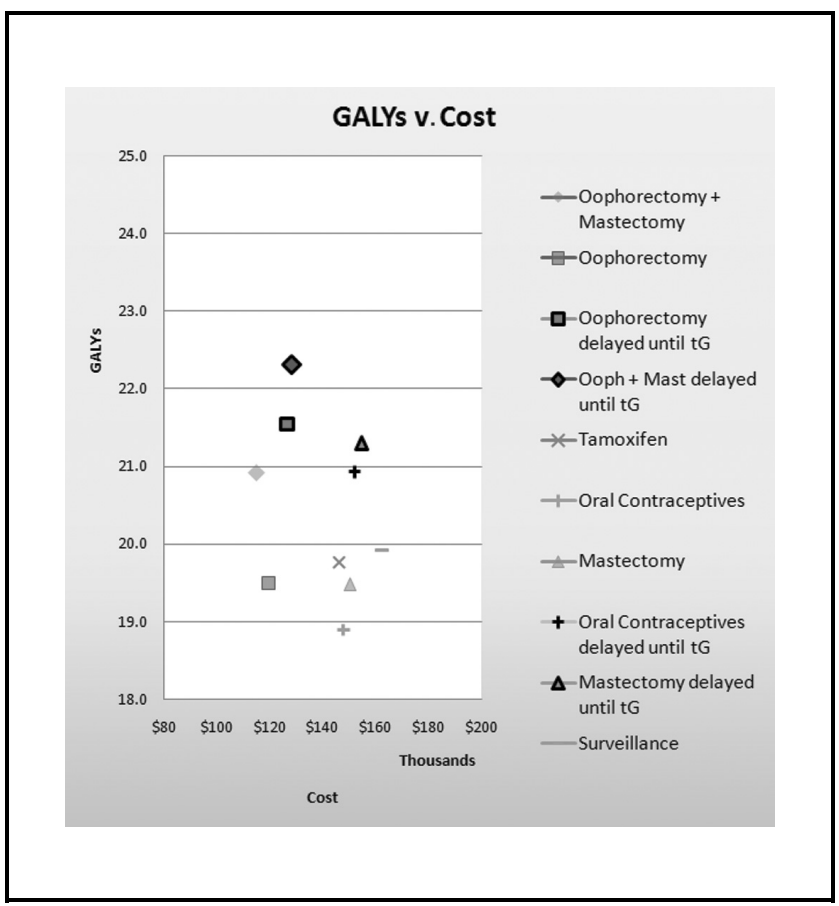

Figure 7 The cost/goal-achieved life year (GALY) analysis corresponding to the cost/life year (LY) analysis of Figure 6. Here the extrinsic goal is to remain fertile for $t_{G}=2$ years, and the goal importance weight is $k_{G}=3$ years (goal achievement is worth 3 years of life). Strategies receive lower $G A L Y$ values to the degree that they impede goal achievement.

The types of extrinsic goals considered here are particularly simple, consisting only of yes/no levels of achievement. Intermediate levels of partial achievement might be appropriate in some situations; however, one would then need to assess utilities for these intermediate levels, an additional burden that would not be feasible in many cases.

Other refinements of extrinsic goal modeling have not been considered here but may be appropriate. One issue is that when an individual achieves an extrinsic goal, she or he would likely soon thereafter aspire to yet other extrinsic goals. In our BRCA model, a woman may want to remain fertile long enough to have a baby but afterwards would no doubt want to live long enough to raise it. Should such potential future extrinsic goals be incorporated into an analysis? No one asks this question for QALYs, but it is relevant there as well-should the possibility of altered future quality coefficients be accounted for? What are the probabilities associated with such altered preferences, for either ongoing or extrinsic goals? Such refinements could considerably complicate model formulation, and we currently know of no easy workarounds. Similarly, one could inquire about the interaction of extrinsic goal achievement with subsequent quality of health. Does extrinsic goal achievement boost or deflate subsequent quality?

Despite its limitations, the QALY model has become the standard for modeling patient preferences in medical decision analyses. The extrinsic goal model presented here constitutes a fundamental augmentation of this standard and has, we believe, the potential to benefit society by substantially broadening the types of patient and community preferences included in these analyses.

\section{ACKNOWLEDGMENTS}

This research was supported by grant number SES0451672 from the National Science Foundation to 
Northwestern University and the University of Illinois at Chicago. We thank 2 anonymous reviewers for their constructive criticism and insightful suggestions.

\section{REFERENCES}

1. Pliskin JS, Shepard DS, Weinstein MC. Utility functions for life years and health status. Operations Res. 1980;28:206-24.

2. Gold MR, Patrick DL, Torrence GW, et al. Identifying and valuing outcomes. In: Gold MR, Siegel JE, Russell LB, Weinstein MC, eds. Cost-Effectiveness in Health and Medicine. Oxford, UK: Oxford University Press; 1996.

3. Tsevat J. What do utilities measure? Med Care. 2000;38(suppl II):II-160-4.

4. Hazen GB. Adding extrinsic goals to the QALY model. Decis Anal. 2007;4:3-16.

5. Schwartz A, Hazen GB, Leifer A, Heckerling PS. Life goals and health decisions: what will people live or die for? Med Decis Making. 2008;28:209-19.

6. Miyamoto JM, Wakker PP, Bleichrodt H, Peters HJM. The zerocondition: a simplifying assumption in QALY measurement and multiattribute utility. Manage Sci. 1998;44:839-49.

7. Sutherland HJ, Llewellyn-Thomas H, Boyd NF, Till JE. Attitudes towards quality of survival: the concept of maximal endurable time. Med Decis Making. 1982;2:299-309.

8. Dolan P, Stalmeier P. The validity of time trade-off values in calculating QALYs: constant proportional time trade-off versus the proportional heuristic. J Health Econ. 2003;22:445-58.

9. Stalmeier PFM, Chapman GB, de Boer AGEM, van Lanschot JJB. A fallacy of the multiplicative QALY model for low-quality weights in students and patients judging hypothetical health states. Int J Technol Assess Health Care. 2001;17:488-96.
10. Miyamoto JM, Eraker SA. A multiplicative model of the utility of survival duration and health quality. J Exp Psychol Gen. 1988;117:3-20.

11. van der Pol M, Shiell A. Extrinsic goals and time trade-off. Med Decis Making. 2007;27:406-13.

12. Schwartz A, Hazen GB, Leifer A, Heckerling PS. Development of goal-sensitive health-related utility assessment procedures. Med Decis Making. In press.

13. Auger RG, Weibers DO. Management of unruptured intracranial arteriovenous malformations: a decision analysis. Neurosurgery. 1992;4:561-9.

14. Hazen GB. Stochastic trees: a new technique for temporal medical decision modeling. Med Decis Making. 1992;12:163-78.

15. Hazen GB. Factored stochastic trees: a tool for solving complex temporal medical decision models. Med Decis Making. 1993;13:227-36.

16. Hazen GB. Stochastic trees and the StoTree modeling environment: models and software for medical decision analysis. J Med Systems. 2002;26:399-413.

17. Anderson K, Jacobson JS, Heitjan DF, et al. Cost-effectiveness of preventive strategies for women with a BRCA1 or a BRCA2 mutation. Ann Intern Med. 2006;144:397-406.

18. Ibrahim JG, Chen M-H, Sinha D. Bayesian Survival Analysis. New York: Springer; 2001.

19. Surveillance, Epidemiology, and End Results (SEER) Program (www.seer.cancer.gov) SEER*Stat Database: Incidence-SEER 17 Regs Limited-Use, Nov 2005 Sub (1973-2003 varying)—Linked to County Attributes-Total U.S., 1969-2003 Counties, National Cancer Institute, DCCPS, Surveillance Research Program, Cancer Statistics Branch, released April 2006, based on the November 2005 submission. 